\title{
Arabische Verfassungen und das Problem der "islamischen Menschenrechte"
}

\author{
Von Hans-Georg Ebert
}

Die überwiegende Mehrheit der arabischen Länder verfügt heute über eine Verfassung oder ein verfassungsähnliches Dokument als grundlegenden Rechtsakt für die Organisation und Struktur des Staates sowie dessen Verhältnis zu șeinen Bürgern. ${ }^{1}$ Der Islam als die vorherrschende Form des religiösen Massenbewußtseins in der Region Nordafrikas und des Vorderen Orients tangiert daher zwei Problemkreise:

1. Die Frage nach der Kompatibilität von Islam und Verfassung angesichts der Anerkennung des Koran als göttliche Offenbarungsschrift und

2. Die Frage nach der verfassungsrechtlichen und verfassungspraktischen Widerspiegelung von islamischen Prinzpien und Normen.

Dieser Beitrag soll sich mit Aspekten des zweiten Problemkreises beschäftigen, der ja letzten Endes aus der Anerkennung einer grundsätzlichen Kompatibilität von Islam bzw. $\check{s}_{\text {ari }}{ }^{c} a$ (Islamisches Recht) und Verfassung resultiert.

Bei der konkreten Analyse der staatlichen Grundgesetze Nordafrikas und des Vorderen Orients kristallisieren sich zwei Schwerpunkte heraus:

1. Die Verankerung von Grundrechten der Bürger und

2. Die Verwirklichung der Volkssouveränität.

Beide Aspekte verdienen insofern besondere Beachtung, als daß sie als grundlegende Elemente einer demokratischen und liberalen Ordnung ihre Wurzeln in der westlich-christlichen Kultur haben, aber im Gefolge exogener und endogener bürgerlicher Transformationen auch die islamische Welt in spezifischer Art und Weise prägen. Inwieweit sich dies verfassungsmäßig niederschlägt, zeigt sich nicht nur in den aktuellen Verfassungsdokumenten, sondern auch in den Bemühungen von islamischen Gremien und Organisationen sowie von einzelnen islamischen Rechtstheoretikern, ein für die gesamte islamische Welt gültiges Grundgesetz zu erstellen. Der Entwurf einer "Islamischen Verfassung", der im

1 Eine Übersicht über die gültigen Verfassungsdokumente (Stand: 1995) der Länder der Arabischen Liga findet sich in: Baumann, H. / Ebert, M. (Hrsg.), Die Verfassungen der Mitgliedsländer der Liga der Arabischen Staaten (vgl. auch die Buchrezension von Ph. Kunig in VRÜ 30 [1997], 268). Berlin 1995. Eine Textübersicht in arabischer Sprache (einschließlich historischer Dokumente und Veränderungen) bietet Huri, Y.Q., Ad-dasatir fi l- ${ }^{\mathrm{c}}$ alam al- ${ }^{\mathrm{c}}$ arabi (nusus wa-ta ${ }^{\mathrm{c}}$ dilat) 1839-1987. Beirut 1989. 
Jahre 1978 von der "Akademie für Islamische Forschungen" an der Kairoer Azhar-Universität verabschiedet und im Jahre 1979 veröffentlicht wurde, ${ }^{2}$ widmet sich in den Kapiteln 4 (Art. 28-43) und 7 (Art. 83-84) dieser Problematik. Dabei werden die Grundrechte und die Volkssouveränität als Bestimmungen, die sich aus der islamischen $\check{s}$ aric ${ }^{c} a$ ergeben, interpretiert und festgeschrieben. Im folgenden soll daher untersucht werden, auf welchen Regelungen das islamische Verständnis von Menschenrechten und Volkssouveränität basiert.

\section{Menschenrechte im islamischen Kontext}

Menschenrechte als individuelle Rechte des Menschen, die diesem in seiner Eigenschaft als natürliches Wesen zustehen und die vom Staat nicht angetastet werden dürfen, können eine unterschiedliche Rechtfertigung erfahren - durch den göttlichen Willen, die Natur oder die Vernunft. Die These von der natürlichen Gleichheit der Menschen wird heute - zumindest verbal - von vielen Muslimen akzeptiert, auch wenn - wie L. Kühnhardt formuliert - "die Verbreitung der Menschrechtsidee nicht automatisch Klarheit über ihr inhaltliches Konzept bewirkt hat". ${ }^{3}$ Die arabischen Termini huquq al-insan oder huquq insaniya für Menschenrechte konnotieren diesen Sachverhalt.

Die verfassungsrechtliche und gesetzliche Verankerung der Menschenrechte in Gestalt der Grundrechte (huquq asasiya oder huquq 'amma) verleiht diesen einen rechtsverbindlichen Charakter. Die Grenzen bei der Wahrnehmung von Grundrechten ergeben sich aus der Achtung der Grundrechte des anderen. Sie sind z.T. in Gesetzesvorbehalten niedergelegt.

Im Gegensatz zu den allen Menschen zustehenden Grundrechten haben nur die Staatsbürger eines Landes Grundrechte im Sinne von Bürgerrechten (huquq madaniya) inne. Das jeweilige Staatsangehörigkeitsgesetz (qanun al-ginsiya) fixiert den Personenkreis, dem diese Bürgerrechte zustehen. Persönlichkeitsrechte (huquq šahsiya) als Rechte des einzelnen "auf Achtung seiner Würde und seines Eigenwertes als Person" ${ }^{4}$ begründen subjektive Abwehrmöglichkeiten gegen Eingriffe in das Privatleben und in die Intimsphäre.

In arabischer Sprache veröffentlicht in Magallat al-azhar. Al-Qahira 51/1979, S. 151-159. Übersetzung und Kommentar: $\mathrm{Al}$-Ghazali, $M$., Introduction to a Draft Islamic Constitution. In: Islamic Studies, Islamabad XX (1981) 2, S. 153-168. Zu weiteren Entwürfen "islamischer Verfassungen" vgl. Badry, R., Mustafa Kamal Wasfi und der Traum einer "islamischen Verfassung". In: Gottes ist der Orient. Gottes ist der Okzident. Festschrift für A. Falaturi zum 65. Geburtstag. Hrsg. von U. Tworuschka, Köln, Wien 1991, S. 83-107, und Müller, L., Islam und Menschenrechte. Sunnitische Muslime zwischen Islamismus, Säkularismus und Modernismus. Hamburg 1996, S. 119121.

Kühnhardt, L., Stufen der Souveränität. Staatsverständnis und Selbstbestimmung in der "Dritten Welt". Bonn, Berlin 1992, S. 244. 
Trotz dieser im wesentlichen unstrittigen Definitionen und der faktischen Akzeptanz der These von der natürlichen Gleichheit der Menschen: Diese These ist nicht der Ausgangspunkt islamischer Theorien. Die absolute Souveränität gehört nach islamischer Auffassung Allah, der Mensch hat in jedem Falle das ewig und überall gültige göttliche Gesetz zu befolgen. Die Durchsetzung des Islam und damit der $\check{s}^{2} i^{c} a$ in der Welt insgesamt avanciert zum eigentlichen Ziel muslimischer Aktion. Diese islamischen Grundwerte erlauben eine Reihe von Folgerungen:

1. Ein unterschiedlicher Ausgangspunkt in der Menschen- und Grundrechtsdiskussion muß nicht notwendigerweise zu unterschiedlichen praktischen Standpunkten führen, wiewohl es voneinander abweichende Haltungen geben kann;

2. Die Spezifik "islamischer" Menschenrechtsauffassungen resultiert aus einigen $\check{s}^{2} i^{c} a$ Regelungen, deren tatsächliche Realisierung zu untersuchen ist;

3. Obgleich die $\check{s} \operatorname{ari}^{c} a$ für die Muslims unveränderbares, weil göttliches Gesetz darstellt, können veränderte gesellschaftliche Bedingungen die reale Wirkung der Bestimmungen beeinflussen (vgl. etwa die faktische Bedeutungslosigkeit des Sklavenrechts);

4. Das Islamische Recht verfügt über ein zwar eingeschränktes, aber dennoch anwendbares Instrumentarium, um auch in bezug auf die Menschen- und Grundrechte rechtsschöpferisch tätig zu werden;

5. Defizite bei der Durchsetzung von Menschen- und Grundrechten zeigen sich im übrigen nicht nur in den islamischen Ländern und können folglich nicht einseitig und apodiktisch eingefordert werden.

\section{a) Die Gleichberechtigung zwischen den Geschlechtern}

Die Aussagen in Koran und Sunna (Überlieferung des Propheten; niedergelegt in den ahadit) zu den Beziehungen zwischen den Geschlechtern betreffen

1. Die Gleichheit von Mann und Frau als menschliche Wesen, die in moralischem und religiösem Sinne die gleiche göttliche Wertschätzung genießen. All_h wendet sich mit seiner Botschaft an alle Menschen, nicht nur an die Männer. Im Koran 49:13 heißt es: "O ihr Menschen, siehe, wir erschufen euch von einem Mann und einer Frau und machten euch zu Völkern und Stämmen...". Neben weiteren diesbezüglichen Koranversen (3:193, 4:36) und entsprechenden Traditionen ${ }^{5}$ finde dies - so M.I. Bagdadi - in der prinzipiellen Anerkennung gleicher Rechte und Pflichten seinen Ausdruck: "Laha min al-haqq ma lahu, calaiha ma ${ }^{c}$ alaihi". ${ }^{6}$ Eine solche Interpretation widerspricht jedoch - wie noch zu zeigen ist - den konkreten rechtlichen und gesellschaftlichen Bestimmungen.

Vgl. al-'Inani, I.M., Huquq al-insan fi š-šaric a al-islamiya. In: Mu'tamar haula dirasat fi ba ${ }^{c} d$ annuzum al-qanuniya al-haliya fi misr. I ${ }^{\mathrm{c}}$ dad: A. Gami ${ }^{\mathrm{c}}$, al-Q_hira 1982, S. 18-22 und 57-58.

Bagdadi, M.I., Huquq al-mar'a al-muslima fi l-mugtamac al-islami. Ar-Ribat 1991, S. 104. 
2. Die physischen und psychischen Unterschiede zwischen Mann und Frau, insbesondere die natürlich-biologische Funktion der Frau als Mutter, werden von den islamischen Gelehrten als wesentliches Element für eine spezifische Rolle der Frau in der Gesellschaft gewertet. $^{7}$ Sie leiten aus der biologischen Ungleichheit eine rechtliche und soziale Ungleichheit ab, die in den Koranversen 2:228 ("doch haben die Männer den Vorrang vor ihnen": wa li-r-rigal calaihinna daragatun) und 4:38 ("Die Männer sind den Frauen überlegen wegen dessen, was Allah den einen vor den anderen gegeben hat, und weil sie von ihrem Vermögen [für die Frauen] auslegen.") deutlich werde. M.I. al-Qaisi verweist auf zahlreiche weitere Koranstellen sowie ahadit unter der Rubrik "al-ibtila' bi-l-banat" (im Sinne einer Prüfung des Mannes durch die Frauen) und "aš-šs'm min al-mar'a" (das Unheil, welches die [untugendhafte] Frau anrichtet). ${ }^{8}$

Der offenkundige Widerspruch zwischen beiden Aspekten erlaubt vielfältige Interpretationen und unterschiedliche Ansichten, indem die eine oder die andere Seite betont und ausgestaltet wird. Gleichsam gelten die koranischen Aussagen und die unzweifelhaften Traditionen in ihrer Gesamtheit als sakrosankt und können daher nicht durch menschliche Entscheidungen außer Kraft gesetzt, wohl aber ihre Anwendung dahingehend geprüft werden, inwieweit die Schaffung bestimmter gesellschaftlicher Bedingungen Voraussetzung für deren Wirken ist. Auf diese Weise kann beispielsweise die geforderte Gleichbehandlung der Ehefrauen durch den Ehemann als ein gültiges Prinzip die Einschränkung oder gar Abschaffung der Polygynie legitimieren.

Wenn islamische Autoren physische und psychische Faktoren als Basis einer göttlich bestimmten Ungleichheit zwischen Mann und Frau bewerten, ${ }^{9}$, so kategorisieren sie keineswegs nach einer generellen Höher- bzw. Minderwertigkeit oder aber einer Bevorteilung bzw. Benachteiligung. Im Gegenteil - Schamgefühl (haya), weibliche Schwäche und weibliche Ehre seien Werte, die eine geschlechterspezifische Rolle der Frau rechtfertig ${ }^{-}$ ten. ${ }^{10}$ Die natürliche Unterscheidung (tafriqa tabiciya) zwischen Mann und Frau bringe notwendigerweise Unterschiede in wichtigen gesellschaftlichen und privaten Bereichen mit sich, so im Hinblick auf

- wirtschaftliche Leistungsfähigkeit;

- religiöse Pflichterfüllung (etwa: menstruationsbedingte Gebetspausen, Einschränkungen bei der Wallfahrt und beim Fasten);

7

Zu den Unterschieden zwischen Mann und Frau zählen sie Körperbau (takwin), Talent (mauhiba) und Fähigkeiten (isti ${ }^{c}$ dadat). Dazu in bezug auf Saudi-Arabien: Vagt, H., Die Frau in Saudi-Arabien zwischen Tradition und Moderne. Berlin 1992, S. 137. Al-Qaisi, M.I., Al-mar'a al-muslima baina igtihadat al-fuqaha' wa-mumarasat al-muslimin. ArRibat 1991, S. 139-153.

9 Mutahhari, M., The Rights of Women in Islam. Teheran 1981, S. 173-174.

10 Forstner, M., Islam und Demokratie. CIBEDO-Texte Nr. 9/10. Köln 1981, S. 16. 
- familiäre Aufgabenverteilung sowie

- strafrechtliche (hudud, qisas und $t a^{c}$ zir), zeugnisrechtliche (šahada), erbrechtliche (mirat $)$ und nicht zuletzt familienrechtliche Wirkungen (Problemkreise: Verschleierung, Polygamie, Geburtenkontrolle, Scheidung). ${ }^{11}$

Die islamischen Auffassungen zur rechtlichen und nichtrechtlichen Rolle der Frau in der Gesellschaft können im konkreten erheblich differieren. So bestreiten puritanische Gelehrte das Recht und die moralische Rechtfertigung für die Frau, einer Erwerbstätigkeit an der Seite des Mannes nachzugehen, wenden sie sich gegen eine gleichberechtigte und gleiche Bildung für Jungen und Mädchen, für Studentinnen und Studenten, leugnen sie die islamische Legitimität der Geburtenkontrolle. ${ }^{12}$ Die geschlechterspezifische Rollenverteilung (infisal) ist bis heute ein wesentliches Charakteristikum islamischen Familien- und Gesellschaftslebens. Ihre Normen betreffen die Einschränkung der Handlungsfähigkeit der Frau außerhalb des eigenen Hauses ebenso wie praktische Verhaltensgebote, etwa im Umgang mit Fremden oder im Kontakt mit Medizinern (bei Geburt oder Krankheit) innerhalb oder außerhalb einer medizinischen Einrichtung. ${ }^{13}$

\section{b) Religionsfreiheit versus Staatsreligion}

Die Genesis des Islam als einer Staats- und Gesetzesreligion und der Prozeß der Normierung in den Rechtsschulen begründen zum einen den Anspruch auf eine Sonderrolle der islamischen Religion und des Islamischen Rechts in Abgrenzung zu anderen Religionsgemeinschaften, zum anderen stellt sich die Problematik der Gleichheit der Menschen und ihrer (gleichen) Verantwortlichkeit vor dem (irdischen) Gericht.

Der Terminus Staatsreligion (din ad-daula) oder offizielle Religion (din rasmi) ist in den aktuellen Verfassungsdokumenten der Länder Nordafrikas und des Vorderen Orients (mit Ausnahme Syriens und Libanons) expressis verbis verankert oder wird als gewohnheitsrechtliches Prinzip respektiert. ${ }^{14}$ Insofern ergibt sich in der arabischen Welt ein weitgehender Konsens hinsichtlich einer grundsätzlichen Festschreibung der besonderen Rolle der

11

12

13

14

Vgl. Bagdadi, M.I., H uq_q al-mar'a al-muslima..., a.a O., S. 127.

Ibn Baz, ${ }^{C}$ A. al- ${ }^{c}$ A.I. ${ }^{c}$ A., Magmu ${ }^{c}$ at al-fatawa wa-r-rasa'il an-nisa'iya. Al-Qahira 1989, S. 14-37.

Vgl. Weber, M., Der kranke Muslim in Deutschland, in: Muslime im Krankenhaus. Hrsg. von $K$. Richter, Altenberge 1983, S. 12-19.

Ebert, H.-G., Die Interdependenz von Staat, Verfassung und Islam im Nahen und Mittleren Osten in der Gegenwart. Frankfurt a. M., Bern, New York, Paris 1991, S. 130-137. 
islamischen Religion bzw. eines - wie M. Forstner formuliert - "numerus clausus der Religionen" ${ }^{15}$ mit personenrechtlich relevanten Folgerungen.

1. Die Gemeinschaft der Muslims genießt gesetzlich bestimmte oder gewohnheitsrechtliche Privilegien, die direkt oder indirekt Auswirkungen auf die Rechtsverhältnisse der islamischen Staaten haben. Direkte Konsequenzen betreffen beispielsweise den Ausschluß von Nichtmuslims von der gesetzlichen Erbfolge, das Eheverbot einer Muslimin mit einem Nichtmuslim, indirekte Konsequenzen resultieren aus der Einschränkung von Bürgerrechten (etwa bei der Bildung von Organisationen und Parteien, bei der Wahrnehmung der Versammlungsfreiheit unter Beachtung der religiösen Gepflogenheiten eines Landes) sowie aus der Vergabe öffentlicher Ämter nach religiösen Gesichtspunkten. ${ }^{16}$ Darüber hinaus sind einzelne Bestimmungen in verschiedenen arabischen Ländern zu beachten, beispielsweise der aus Art. 10/h des libyschen "Gesetzes Nr. 18 von 1980 über die Bestimmungen des Staatsbürgerschaftsgesetzes" 17 abgeleitete Verlust der arabischen (d.h. der libyschen Staatsbürgerschaft) bei "Abfallen" von der islamischen Religion (irtidad).

2. Der "islamische" Staat erklärt sich selbst zum Sachwalter des Islam und fühlt sich berufen, in islamischen Angelegenheiten letzten Endes verbindlich zu entscheiden und die Rechts- und Religionsgelehrten in ihrer Tätigkeit zu kontrollieren. Während die erzwungene Einhaltung der ${ }^{c}$ ibadat und die öffentlich geförderte Propagierung des Islam nach innen und außen $\left(d a^{c} w a\right)$ ein staatliches Legitimationsbedürfnis befriedigen, greift die faktische "Verbeamtung" der Rechts- und Religionsgelehrten ('ulama') unmittelbar in die Substanz der Rechtsverwirklichung ein, indem diese als vom Staat bestellte Richter und andere Amtsträger in der Judikative beauftragt sind, das staatlich-gültige Recht durchzusetzen. Dieser Prozeß verläuft keineswegs harmonisch, sondern in ständiger Auseinandersetzung um den Handlungsspielraum der ${ }^{c}$ ulama'.

3. Der Begriff Staatsreligion konnotiert die besondere Rolle der $\check{s}^{a}{ }^{c} a$ im Rechtsetzungsprozeß. Dabei sind zwei wesentliche Aspekte zu berücksichtigen:

a) Die Übereinstimmung der erlassenen Gesetze, Verordnungen und sonstigen Rechtsakte mit den Vorschriften des Islam und

b) Die šarica (bzw. der fiqh) als eine oder die Quelle (Hauptquelle) der Gesetzgebung. Eine solche Formulierung findet sich in zahlreichen arabischen Verfassungsdokumenten.

Forstner, M., Das Menschenrecht der Religionsfreiheit und des Religionswechsels als Problem der islamischen Staaten. In: Kanon. Jahrbuch der Gesellschaft für das Recht der Ostkirchen, Wien 10/1991, S. 108.

$16 \mathrm{Vgl}$. beispielsweise die Praxis der Regierungsbildung in Ägypten.

17

Al-garida ar-rasmiya.Tarabulus 8.12.1980, S. 857-862. 
Die eigentliche Problematik der Aussage betrifft vor allem das islamische Strafrecht, welches eine Reihe drastischer Körperstrafen für hudud-Delikte vorsieht (Steinigung, Kreuzigung, Abtrennung von Gliedmaßen, Auspeitschen) und das Prinzip der Talion (qisas) verankert.

Die Staatsreligion im islamischen Kontext kann nicht mit der Vorstellung einer Theokratie (daula diniya) gleichgesetzt werden. Die überwiegende Mehrheit muslimischer Gelehrter favorisiert einen "zivilen" Staat (daula madaniya), der auf der šaric ${ }^{c} a$ basiert und Verantwortung für die Religion trägt, nicht jedoch im Sinne einer Theokratie von einem göttlichlegitimierten Herrscher oder vom "islamischen Klerus", der ja im Verständnis des Islam ohnehin nicht existiert, regiert wird. ${ }^{18}$

Die Anerkennung des Islam als Staatsreligion widerspricht zwar einer generellen Gleichsetzung der Religionen, verhindert jedoch nicht grundsätzlich die Religionsfreiheit. ${ }^{19}$ Religionsfreiheit schließt Glaubensfreiheit als innere Seite und Bekenntnisfreiheit als äußere Seite ein. ${ }^{20}$ Die in den modernen Verfassungsdokumenten verwendeten Termini für Religionsfreiheit (hurriyat al-adyan), Glaubensfreiheit (hurriyat al-caqida, hurriyat al$m u^{c}$ taqad, hurriyat al-ictiqad) und Bekenntnisfreiheit - als Kultusfreiheit - (hurriyat mumarasat $a \check{s}-\check{s ̆}^{c} a^{\prime} i r$ ad-diniya, hurriyat al-qiyam bi-ša $a^{c} a^{\prime} i r$ al-adyan, hurriyat mumrasat aš-šu'un ad-diniya, hurriyat al-qiyam bi-gami ${ }^{c}$ šs-ša $a^{c} a^{\prime} i r$ ad-diniya, hurriyat al-qiyam bi$\check{s} a^{c} a^{\prime} i r$ ad-din) orientieren sich an westlichen Vorbildern, werden aber letzten Endes auf koranische Wurzeln (usul) zurückgeführt (vgl. Koran 2:257, 16:126).

Die Religionsfreiheit in den arabischen Ländern muß - was ihre theoretische und praktische Seite anbelangt - stets im Kontext des Islamischen Rechts erfaßt werden, obwohl sich dies verfassungsmäßig nur z.T. widerspiegelt. Daraus folgt, daß nur die Anhänger der Buchreligionen (ahl al-kitab), also in erster Linie Christen und Juden, ${ }^{21}$ ihre religiösen

Krämer, G., Islam, Menschenrechte und Demokratie. In: Weltmacht oder Chaos? Beiträge zur internationalen Politik. Festschrift zum 75. Geburtstag von Professor Dr. K. Ritter. Hrsg.: A. Zunker, Baden-Baden 1993, S. 340; Zubaida, S., Islam, the State and Democracy. Contrasting Conceptions of Society in Egypt. In: Middle East Report, Washington D.C. November-December 1992, S. 4-5. Eine gewisse Ausnahme bildet die (schiitische) Staatskonzeption Khomeinis (gest. 1989), der die 'ulama' und fuqaha' in der Zeit der Abwesenheit des Imams als zur Führung des Staates ermächtigt perzipiert.

Ähnliches gilt auch für europäische Länder, beispielsweise für Italien bis zum Konkordat vom 18. 2. 1984. Avenarius, H., Recht von.... a.a.O., S. 416.

21 Auch Zaradušt (Zoroastrier) und Sabi'a (Sabier). Letztere werden beispielsweise im Irak als Buchreligion respektiert. Eigentlich handelt es sich um Mandäer, die dadurch den Schutz einer "Buchreligion" genießen. Vgl. Rudolph, K., Researches on the Relation between Mandaens and Manichaens after the Discovery of the Cologne Mani-Codex (CMC). Vortrag auf der ICANASKonferenz. Hongkong 1993, S. 3. 
Pflichten wahrnehmen können - vorausgesetzt, sie anerkennen die muslimische Souveränität, entrichten ihre Steuern und beteiligen sich nicht an Kämpfen gegen Muslime. ${ }^{22}$ Andererseits haben Ungläubige (kuffar), Polytheisten (mušrikun) und sonstige Ketzer (mulhidun) kein Recht, sich zu ihren Auffassungen zu bekennen. Sie sind zwangsweise zu bekehren. Religionswechsel wird lediglich als eine Möglichkeit angesehen, den Islam anzunehmen, nicht aber umgekehrt sich einer anderen Religionsgemeinschaft anzuschließen oder gar sich als Atheist zu bekennen. Der murtadd (Apostat) soll gemäß Koran 2:214, 4:136 und 4:137 mit dem Tode bestraft werden, obwohl sowohl die Strafzumessung als auch die Klassifizierung als hadd-Strafe strittig sind.

Die Koexistenz von Religionsfreiheit und Beschränkung auf Buchreligionen stellt sich als ein innerer Widerspruch dar, der jedoch - wie auch im Verhältnis von Staatsreligion und Religionsfreiheit - vor allem ein Problem der praktischen Möglichkeiten nichtislamischer religiöser Gemeinschaften und Individuen ist, gesellschaftlich gleichberechtigt zu agieren. Die jüngste Vergangenheit beweist, daß das Phänomen der Religionsfreiheit von muslimischer Seite stärker als (innere) Glaubensfreiheit denn als (äußere) Bekenntnisfreiheit verstanden wird. Tendenziell äußert sich das in der geforderten und geförderten Anwendung des islamischen Personalstatuts auch auf Nichtmuslime.

Die erläuterten islamischen Prinzipien der geschlechter- und religionsspezifischen Ungleichheit widersprechen in der Tat westlichen Grundrechtsauffassungen. Das bedeutet jedoch nicht, daß es nicht von islamischer Seite Bemühungen und Möglichkeiten gibt, Menschenrechte als mit der $\check{s} \operatorname{ari}^{i} a$ vereinbar zu deklarieren bzw. diese als islamisch-originär zu begründen. Die theoretischen Ansatzpunkte liegen vor allem in folgenden Elementen:

a) Einige islamische Theoretiker betonen die ethischen Werte des Islam und beschreiben die Prinzipien Freiheit (hurriya), Beratung ( $̌$ ura - s.u.) und Gerechtigkeit ( ${ }^{c} a d l$ ), wie das beispielsweise der ägyptische Publizist $M$. ${ }^{~}$ Ammara tut. ${ }^{23}$ An diesen Prinzipien seien staatliche Maßnahmen zu messen. Hier liege der eigentliche Kristallisationspunkt von Menschenrechten. Die besonders im Maghreb intensiv geführte Diskussion um die maqasid $a \check{s}$-šaric a ("Intentionen" des Islamischen Rechts), die auf den andalusischen Gelehrten A.I.I. aš-Šatibi (gest. 1388) zurückgeführt werden, widerspiegelt die Tendenz der Ethisierung des Islamischen Rechts.

b) Bei der islamischen Begründung von Menschenrechten kommt der Sunna eine Schlüsselrolle zu. Zugleich - so fordern "aufgeklärte" Muslime - müsse die islamische Tradition 
von all demjenigen Ballast befreit werden, der über Jahrhunderte die eigentliche islamische Botschaft deformiert habe. Diese Art Fundamentalismus (nicht im Sinne des Islamismus) eröffnet Möglichkeiten für eine historische und kritische Sicht der Quellen.

c) Die Erstarrung des islamischen Rechts müsse durch eine echte Wiederbelebung des igtihads, d.h. durch die Wiedereröffnung seines Tores, überwunden werden. Die selbständige rechtsschöpferische Tätigkeit könne prinzipiell neue Ansatzpunkte schaffen.

\section{Volkssouveränität und šura}

Islamische Staatsauffassungen beschäftigen sich vor allem mit dem Verhältnis von hukm (im Sinne einer Entscheidung des einzelnen; auch als hukm fardi bezeichnet) und šura (Beratung). Die šura gilt dabei nicht als gleichberechtigtes, sondern als ein dem hukm untergeordnetes Prinzip. In den "modernen" Staatstheorien muslimischer Theoretiker wird das Verhältnis von hukm und šura folgendermaßen charakterisiert:

1. Šura als Grundlage für die Ausübung des hukm im Islam (zusammen mit Gerechtigkeit und Freiheit - asas mumarasat al-hukm fi l-islam); ${ }^{24}$

2. Šura als Prinzip des islamischen Regierungssystems (zusammen mit Gerechtigkeit, Gleichheit, Freiheit und Verwantwortung - mabda' nizam al-hukm al-islami); ${ }^{25}$

3. Šra als Prinzip des islamischen hukm ( $q a^{c} i d a$ oder asas al-hukm al-islami). ${ }^{26}$

Einige Verfassungsdokumente in Nordafrika und im Vorderen Orient nehmen direkt oder indirekt auf die šura Bezug. Sowohl hukm als auch šura werden auf die šaric a zurückgeführt, erscheinen somit als ewig und unverzichtbar. Die 42. (mekkanische) Sure des Koran trägt den Namen "aš-šura". Insbesondere Koran 42:36 ("und die auf ihren Herrn hören und das Gebet verrichten und ihre Angelegenheiten in Beratung untereinander [erledigen]...") und 3:153 ("Drum vergib ihnen und bete für sie um Verzeihung und ziehe sie zu Rate in der Sache...") gelten als autoritative Belege für die Praktizierung der šura. ${ }^{27}$ Auch in der Sunna sowie in verschiedenen grundlegenden islamischen Werken (z.B. Nahg al-balaga) finden sich Hinweise auf die Ausübung der šura. Konkrete Bestimmungen zum Inhalt und zu den Modalitäten der šura fehlen jedoch ebenso wie eine verbindliche, übereinstimmende

24

25

26

27

$S a^{c} i d, S{ }^{c} A$., Al-hakim wa-usul al-hukm fi n-nizam al-islami (as-siyasi wa-l-iqtisadi wa-l-igtima ${ }^{{ }^{c}}{ }_{i}$ wa-l-fikri). Al-Qahira 1985, S. 90.

Al-Ansari, ${ }^{C}$ A. al-H. I., Nizam al-hukm fi l-islam. Qatar 1985, S. 11.

Musa, M.Y., Nizam al-hukm fi l-islam. I. Teil: Al-imama au-riyasat al-umma wa-ma yata ${ }^{\mathrm{c}}$ allaqu biha min buhut. Al-Qahira o.J., S. 177.

Vgl. Waraqa li-l-hiwar. Aš-šura wa-nizam al-hukm. In: Nadwat at-taiyarat al-islamiya al-mu'asira fi l-watan al- ${ }^{\text {c}}$ arabi, Malta 1987, S. 219-229. 
Auffassung bezüglich ihrer Anwendung. Im Kern wir die šura als eine allgemeine Methode oder ein Verfahren, nicht aber als eine konkrete Institution qualifiziert. Die Beratung sei in allen gesellschaftlichen Bereichen anwendbar: Kultur, Wissenschaft, Soziales, Verwaltung, Politik. Ihre Legitimität erwachse sowohl aus der Praxis des Propheten selbst (vgl. die bai ${ }^{c} a$ - Huldigung - von ${ }^{c} A q a b a$ ) als auch aus den staatsrechtlichen Aktivitäten der Prophetengenossen (sahaba) und der Kalifen bzw. - was die Schiiten anbelangt - der Imame.

Die politischen und sozioökonomischen Veränderungen in der islamischen Welt sowie die Intensivierung des Kontakts der Muslime mit europäischen Nichtmuslimen haben die Rückbesinnung auf die "wahren" und "authentischen" Quellen des Islam stimuliert. Dieser Prozeß - vor allem von den Reformdenkern des 19. und 20. Jh. (Afgani, ${ }^{c}$ Abduh, Rida) beeinflußt - mündete in eine Neubestimmung der šura als Ausdruck demokratischer Mitbestimmung im Rahmen der $\check{s} a r i c^{c} a$. Das Spektrum der Befürworter der šura reicht von säkularistischen bis hin zu islamistischen Kräften. Der äußere Konsens bei der Anwendung der šura verdeckt jedoch einen inneren Dissens, der sich in der Hauptsache auf folgende Punkte erstreckt:

1. Die Frage, ob die šura gemäß Koran und Sunna verpflichtenden (wagib) oder empfehlenden (mandub) Charakter trägt;

2. Die Kompetenzen der šura-Gremien hinsichtlich der Kodifizierung und Durchsetzung des Islamischen Rechts und

3. Die Festlegung von Kriterien für die Bezeichnung einer Institution als šura.

Die bereits eingangs erwähnten Bemühungen islamischer Theoretiker, eine für die gesamte islamische Welt gültige Verfassung zu erarbeiten, kulminieren in der grundsätzlichen Akzeptanz der Beratung für den Herrscher. Zwei Beispiele mögen das illustrieren:

a) A.A. Maududi (gest. 1979), der bedeutende Ideologe der "Gamacat-i islami", der pakistanischen Variante der "Muslimbrüder", fordert als "Verfassungsprinzip" Wahl und Konsultation in Übereinstimmung mit den Erfordernissen der Zeit; ${ }^{28}$

b) Der Ägypter I. al-Badawi unterbreitet das "Projekt einer islamischen Verfassung", indem er in insgesamt 30 Artikeln die Modalitäten der šura und die Pflichten des Staatspräsidenten ( $r a^{\prime} i s$ ad-daula) fixiert. ${ }^{29}$

Die Vorstellungen von der šura sind jedoch nicht nur in derartige allgemeine, praktisch irrelevante Verfassungsmodelle geflossen, sie sind heute in zahlreichen islamischen Län-

Vgl. Maudoodi, A.A., Economic and Political Teachings of the Qur'an. In: A History of Muslim Philosophy. Ed. by M.M. Sharif. Bd. 1, Wiesbaden 1963, S. 194-195; Maududi, S.A.A., The Islamic Law and Constitution. Transl. and ed. by Kh. Ahmad. Lahore, Dacca 1969, S. 245-261.

29

Al-Badawi, I., Mabda' aš-šura fi š-šarica al-islamiya. In: Magallat al- ${ }^{c}$ ulum al-idariya, al-Qahira 22 (1980) 2. S. 198-203. 
dern mit konkreten Verfassungsregelungen zu parlamentarischen und/oder Vertretungskörperschaften verbunden. Drei wesentliche Varianten sind zu unterscheiden:

\section{Das parlamentarische Verständnis der ̌̌ura}

Die Verfassung der Islamischen Republik Iran vom 15.11 .1979 (i.d.F. vom 28.7.1989) ${ }^{30}$ benennt das zur Gesetzgebung ermächtigte Gremium als "magles-e šoura-ye eslami" (Art. 62-99). Darüber hinaus bezeichnet das iranische Grundgesetz (Art. 7) alle Räte auf zentraler und örtlicher Ebene (Provinz, Kreis, Stadt, Stadtviertel, Bezirk, Dorf "und dergleichen") als šura-Organe. Die besondere islamische Legitimität des iranischen Parlaments erwächst zudem aus der Mitarbeit von 'ulama', die jedoch nicht als ex officio-Mitglieder agieren, sondern als gewählte Bürger des Landes. Die Souveränität des "magles-e šoura-ye eslami" wird zum einen durch die staatsrechtlich überragende Stellung des "Islamischen Führers" (faqih) gemäß Art. 110 und zum anderen durch die Schaffung eines sog. "Wächterrates", der die Übereinstimmung der angenommenen Gesetze mit den Prinzipien des Islam und der Verfassung zu prüfen hat (Art. 4 und 91-99), und eines sog. Schlichtungsrates (Art. 112), der im Falle von Meinungsverschiedenheiten zwischen Parlament und Wächterrat vermitteln soll, eingeschränkt.

\section{Die šura als beratendes Organ neben dem Parlament als gesetzgebende Institution}

Herausragendes Beispiel für dieses Verständnis der šura ist der ägyptische maglis aš-šura, der gemäß Art. 194-205 der Verfassung der Arabischen Republik Ägypten vom 11.9.1971 (i.d.F. vom 22.5.1980) ${ }^{31}$ mit der Verfassungsänderung vom 22.5.1980 gebildet worden ist. Die Kompetenzen des maglis aš-šura erstrecken sich auf die Diskussion von Gesetzesprojekten und die Beratung von Präsident und Parlament in wichtigen innen- und außenpolitischen Angelegenheiten. Ein Drittel der Mitglieder des Rates wird vom Präsidenten ernannt. Obwohl weder in der Verfassung noch im "Gesetz Nr. 120 von 1980 über den maglis aššura" expressis verbis auf eine "islamische Ausrichtung" dieses Gremiums Bezug genommen wird, legitimieren 'ulama' und Vertreter der koptischen Kirche die Religionspolitik des Staates in Auseinandersetzung mit der islamistischen Herausforderung.

\section{Die šura als beratendes Organ}

Die Beratung des Herrschers durch ausgewählte Notabeln gehört insbesondere in den Golfstaaten zu den Traditionen des politischen Systems. Dies ist nicht direkt an ein staatliches Grundgesetz gekoppelt, kann jedoch durchaus damit in Verbindung stehen. In den

30

Kejhan. Teheran 17.11.1979, S. 1-4, Verfassung der Islamischen Republik Iran. In: Iran und die Islamische Republik. Heft Nr. 6, Bonn, Mai 1980. Verfassungsänderung in: Aktueller Informationsdienst Moderner Orient. Hamburg 17/1989, S. 59-81, Tehran Times, Teheran 3.8.1989.

Dustur gumhuriyat misral-carabiya. Al-Qahira 1985, S. 1-43. 
90er Jahren erlebte diese Konzeption bedingt durch innere und vor allem äußere Veränderungen infolge des Golfkrieges einen bemerkenswerten Aufschwung. Der saudische König verfügte die "Ordnung über den Konsultativrat" (nizam maglis aš-šura) vom 1.3.1992 ${ }^{32}$, ernannte per Dekret am 20.8.1993 die 60 Mitglieder des Rates ${ }^{33}$ und legte ebenfalls per Dekret vom 20.8.1993 die Arbeitsweise des Konsultativrates fest. ${ }^{34}$ Neben der saudischen Grundordnung (an-nizam as-sac $a^{c}$ di li-l-hukm) und der Ordnung über die Provinzen (nizam al-manatiq) - jeweils vom 1.3.1992 - begründen die 30 Artikel der "Ordnung über den Konsultativrat" ein Beratungsgremium, dessen Kompetenzen nach Art. 14 in der Meinungsäußerung zur allgemeinen Politik und der Diskussion von Gesetzesprojekten der Regierung, die jedoch erst durch königliche Order in Kraft gesetzt werden können, liegen. Der Monarch selber betonte, daß der maglis aš-šura auf dem bisherigen Mechanismus der šura auf baue und der Festigung des etablierten Systems diene. ${ }^{35}$

\section{Schlußfolgerungen}

1. Die Widerspiegelung des Islam in den Verfassungen und Grundgesetzen Nordafrikas und des Vorderen Orients ist abhängig sowohl von den Spezifika des religiösen Massenbewußtseins, der religiösen und klerikalen Struktur, den innerstaatlichen politischen Bedingungen und den Traditionen des Rechtssystems als auch von den Intentionen und Auffassungen der politischen Führungskräfte.

2. Der Gradmesser für die tatsächliche Umsetzung islamischer Verfassungsnormen ist in jedem Falle die politisch-staatliche Praxis. Die Kodifikation des Islamischen Rechts avanciert zu einem wesentlichen Kriterium innerer Auseinandersetzungen, die aber generell nicht auf religiöse Ursachen allein zu reduzieren sind.

3. Die Bestimmungen der $\check{s}{ }^{i c}{ }^{c} a$ verhindern keineswegs a priori die Durchsetzung der Menschen- und Grundrechte. Auch im Westen sollte die islamische Religion weniger als ein Hindernis, sondern vielmehr als eine moralisch-ethische Chance verstanden werden. Neue Feindbilder erschweren diesen Prozeß, der ohnehin von Mißverständnissen, Mißtrauen, Vorurteilen, Verzerrungen und Besserwisserei auf beiden Seiten durchsetzt ist.

4. Auch wenn das šura-System innerhalb der $\breve{s} a i^{c} a$ nicht mit den Vorstellungen der Volkssouveränität deckungsgleich ist, so lassen sich mit seiner Hilfe demokratische Maßnahmen islamisch authentifizieren. Der Gedanke des Parlamentarismus kann auf

Text in: Al-magalla. London 4-10 March 1992, No. 630, S. 22-23.

33

Aktueller Informationsdienst Moderner Orient. Hamburg 18/1993, S. 35-36.

34

Aktueller Informationsdienst Moderner Orient. Hamburg 18/1993, S. 34-35.

35

Aktueller Informationsdienst Moderner Orient. Hamburg 6/1992, S. 38. 
diese Weise Verbreitung finden, wobei in einer Gesellschaft, in der einfachste Bedürfnisbefriedigung im Mittelpunkt steht, sozioökonomische Veränderungen unabdingbar sind. Auch hier gilt: Der Islam bietet vielfältige Ansatzpunkte zum Dialog und ist nicht das Hemmnis, für das er im Westen leider immer noch gehalten wird. 
these new political structures are consolidated and whether the constitutional documents are normative models put to practice in reality. The article does not try to give a comparative overview over the constitutions in Latin America, but tries to show some general trends of constitutional developments on the continent.

The author shows that the instrumentalization of constitutions is an expression of autocracy and constitutional symbolism is an expression of democratization, and he goes back to consider the authoritarian military regimes of the sixties and seventies in this regard. Drawing a line between legal text and legal norm, the author notes that the democratization of the past years has led to insufficient effectiveness and a lack of normative force of constitutional instruments. Characteristic for the relationship between law and society in Latin America is the legalistic and severe punishment against the ordinary, 'subintegrated' people on the one hand and the exemption from punishment for the privileged on the other hand one of the roots of the harsh discrimination of certain groups of the society, such as the street children or the Indians. The author considers this gap between legalistic stringency and exemption from punishment as one fundamental obstacle to the implementation of the norms providing rule of law and democracy. He suggests that the existing processes for the integration of all strata of society into public life should be accompanied by criminal punishment and social conditions allowing the identification of the democratic and due process of law and making a corresponding social consensus possible.

\section{Arabian Constitutions and the Issue of "Islamic" Human Rights}

\section{By Hans-Georg Ebert}

The vast majority of Arabian countries have a written constitution or constitutional documents as a foundation for the organization and structure of the state as well as the relationship to the people. The article deals with the influence of Islamic rules and principles on constitutional law and practice in the field of human rights.

The author first introduces into the specific situation of human rights in the Islamic context, then deals with the influence of Islamic rules and principles on the right to equality before the law, the freedom of religion and the doctrine of sovereignty of the people. He concludes that the provisions of Shari'a do not hinder the implementation of human rights per se and, although the doctrine of the sovereignty of the people is not fully compatible with Shari'a, that there is room for a sovereign parliament and thus compatible structures. 